\title{
Clinical-grade human umbilical cord-derived mesenchymal stem cells reverse skeletal muscle dysfunction in age-associated sarcopenia mice
}

Yali Jia ( $\square$ jiayali2016@hotmail.com )

Beijing Institute of Radiation Medicine https://orcid.org/0000-0002-2896-6021

Ailing Wang

Beijing Institute of Radiation Medicine

Chao Wang

Beijing Institute of Radiation Medicine

Jinglei Zhai

Institute of Zoology Chinese Academy of Sciences

\section{Bichun Zhao}

Beijing Institute of Radiation Medicine

\section{Tuling Liao}

Beijing Institute of Radiation Medicine

Ning Cao

920th Hospital of Joint Logistics Support Force

\section{Ruyu Su}

South China Institute of Biomedicine

\section{Lijuan He}

Beijing Institute of Radiation Medicine

\section{Yanhua Li}

Beijing Institute of Radiation Medicine

\section{Wen Yue}

Beijing Institute of Radiation Medicine

\section{Xuetao Pei}

Beijing Institute of Radiation Medicine

\section{Research Article}

Keywords: Human umbilical cord-derived mesenchymal stem cells, Age-associated sarcopenia, Skeletal muscle dysfunction, Satellite cells, Autophagy

Posted Date: March 1st, 2022 
DOI: https://doi.org/10.21203/rs.3.rs-1369361/v1

License: (c) (1) This work is licensed under a Creative Commons Attribution 4.0 International License. Read Full License 


\section{Abstract}

With the expansion of the ageing population, age-associated sarcopenia (AAS) has become a severe clinical disease of the elderly and a key challenge for healthy ageing. However, there are no approved therapies for AAS. In this study, we first used clinical-grade human umbilical cord-derived mesenchymal stem cells (hUC-MSCs) to treat AAS in two classic mouse models (SAMP8 mice and D-galactose-induced ageing mice) and studied the effects at the behavioural, histological, cellular and molecular levels by means of behavioural tests, immunostaining, western blotting and others. Core data results showed that hUC-MSCs dramatically restored skeletal muscle strength and performance in two AAS mouse models via the mechanisms including raising the expression of crucial extracellular matrix proteins, activating satellite cells, enhancing autophagy, and impeding the cellular ageing. For the first time, we comprehensively evaluated and demonstrated the preclinical efficacy of clinical-grade hUC-MSCs in the treatment of AAS in two mouse models. Our study highlights a promising strategy to improve and treat AAS and other age-associated muscle diseases.

\section{Introduction}

Sarcopenia is a progressive and generalised skeletal muscle disorder involving the accelerated loss of muscle mass and function [1, 2], which could be divided into primary sarcopenia and secondary sarcopenia [3]. Primary sarcopenia (or age-associated sarcopenia, AAS) is used to define sarcopenia that is caused by ageing itself; while secondary sarcopenia (or disease-associated sarcopenia, DAS) is caused by diabetes mellitus, cancer, chronic obstructive pulmonary disease, or heart failure [3-5], which also needs appropriate treatment of the underlying disease. With the expansion of the ageing population, the problem of AAS becomes increasingly severe, which seriously impacts the lifestyle of the elderly $[6,7]$. Even though physical exercise is proven to be the most effective method to prevent AAS mouse model, exercise is usually impractical or inefficient for older people with reduced functional capacities. Several clinic pharmaceuticals, such as testosterone, growth factors and dehydroepiandrosterone, were reported to be helpful on AAS, but with controversial effects [8,9]. In fact, there are no approved therapies for AAS. Hence, it is very urgent to generate new treatments for AAS.

Mesenchymal stem cells (MSCs) are widely utilized in cell therapy due to their anti-fibrosis, immunomodulatory properties, and releasing various biologically active molecules [10-12]. It's reported that MSCs could enhance muscular regeneration in animal and cellular models [13-18]. Specifically, human umbilical cord-derived mesenchymal stem cells (hUC-MSCs) not only had a higher yield without the invasive procedures and ethical issues but could secrete a wide range of multifunctional factors [19, 20]. Previously, our group has formed a complete system for obtaining clinical-grade hUC-MSCs according to the current Good Manufacturing Practice (cGMP) guidelines, and the obtained cells fully met the quality criteria of the National Institute of Food and Drug Control (NIFDC) and have been shown to possess good preclinical efficacy in the intervention or treatment of neurodegeneration associated with ageing $[21,22]$. Furthermore, therapeutic effects of hUC-MSCs have been demonstrated on muscular 
atrophy experimental models [23-25], which brings new hope to the treatment for AAS. Therefore, we hypothesized that hUC-MSCs might be a superior source for reversing muscle dysfunction of AAS.

Here, for the first time, we comprehensively evaluated the preclinical efficacy of clinical-grade hUC-MSCs on AAS mouse models, including the SAMP8 mice (a senescence-accelerated mouse commonly used as AAS model) and D-galactose (D-gal)-induced ageing model (a systemic and homogeneous ageing model with the acceleration of senescence), both of which were proven to have the typical phenotype of AAS in our results. Based on the behavioural test, hematoxylin-eosin (H\&E) staining, immunostaining and western blotting, we found that administration of hUC-MSCs could effectively improve muscle strength, restore muscle morphology and performance of ageing skeletal muscle in AAS mouse models via the mechanisms including raising the expression of crucial extracellular matrix proteins, activating skeletal satellite cells, enhancing autophagy, and impeding the cellular senescence. Collectively, our study provided a promising strategy for the prevention and treatment of AAS and other age-associated muscle diseases.

\section{Materials And Methods}

\section{Isolation, cultivation and identifition of hUC-MSCs}

The Ethics Committee approved all procedures involving human subjects in this study at Sun Yat-sen Memorial Hospital, Sun Yat-sen University Guangzhou (Approval number: 2021-01-01), and all the patients gave their written informed consent to participate. Clinical-grade hUC-MSCs were used in this study which was greatly optimized in previous research. The full link, including isolation, cultivation, identification, quality control, and storage, was confirmed to the quality standards. The generation time of hUC-MSCs used in this study was severely restricted from passage 3 to 5 .

\section{Animals}

The SAMP8 and SAMR1 mice were purchased from the Health Science Center of Peking University and could not be tested until they were four months old. Meanwhile, 2-month-old male C57BL/6 mice were used for the study [26]. They are placed in a conventional state, under appropriate temperature and humidity control, 12 hours of light/ 12 hours of the dark cycle, and can freely obtain food and tap water. The Committee approved the Ethics of Animal Experiments of the Academy of Military Medical Sciences, and all animals were cared for following the Guide for the Care and Use of Laboratory Animals.

\section{Treatment}

For the SAMP8 mouse model, the normal group was SAMR1 mice; SAMP8 mice were divided into two groups: (I) P8-PBS group; (II) P8-MSC group, they were injected intraperitoneally with $5 \times 10^{6}$ hUC-MSCs once a week for eight weeks. Accordingly, male C57BL/ 6 mice were divided into two groups at two months: normal and D-gal treatment groups. D-galactose (100 mg/kg, Sigma, USA) was injected subcutaneously every day for eight weeks. At seven months, the latter was further divided into two 
groups: (I) D-gal-PBS group; (II) D-gal-MSC; they were intraperitoneally administered $5 \times 10^{6}$ hUC-MSCs once every two weeks for 12 weeks. After the injection of the above mice, conduct behavioural experiments, then kill the mice, and take gastrocnemius muscle and EDL for follow-up tests.

\section{Rota Rod System}

The experiment included two training days and one test day. Ensure that each mouse is familiar with the experiment after two training days and move stably on the rotating rod. On the day of the test, the mice fell was recorded as the fatigue tolerance time.

\section{Grip test}

Put the mouse in the centre of the metal mesh, grasp its tail and pull it back until the mouse's paws are released. Record the instrument data, i.e. the grip strength of mouse limbs.

\section{Hematoxylin-Eosin staining}

Firstly, the paraffin section baking tray was dewaxed, stained with hematoxylin for 10 minutes, washed with running water, differentiated with $0.7 \%$ hydrochloric acid and ethanol for a few seconds, and then rinsed with tap water; After 15 minutes, dye with 95\% ethanol, alcohol-based eosin, 95\% ethanol (I, II), $100 \%$ ethanol (I, II), xylene and xylene (I, II) for 30 seconds, and finally cover the film.

\section{Immunohistochemical experiment}

Mice were perfused transcardially, followed by $50 \mathrm{ml}$ of $4 \%$ paraformaldehyde, took out their muscle tissue and fixed in 4\% PFA overnight. Each tissue was embedded in paraffin and $5 \mu \mathrm{m}$-thick coronal sections. A standard histological immunohistochemical experiment procedure was performed. Tissue sections were incubated overnight with Monoclonal Anti-Myosin (Skeletal, Fast) antibody (Sigma, Germany, M4276) or Monoclonal Anti-Myosin (Skeletal, Slow) antibody (Sigma, Germany, M8421) or Rabbit polyclonal to Dystrophin (Abcam, USA, ab15277) or Anti-Myosin Heavy Chain Antibody (Sigma, Germany, 05-716). Then the secondary antibody was incubated. The final step is DAB chromogenic reaction. The nucleus of hematoxylin stained is blue, and the positive expression of DAB is brownish yellow. The number and diameter of individual cells were quantified by ImageJ software (version 1.8.0, National Institutes of Health) and Image-Pro Plus (version 6.0.0, media cybernetics).

\section{Immunofluorescent staining}

Gastrocnemius muscle sections were fixed in $4^{\circ} \mathrm{C}$ pre-cooled acetone for 25 minutes and permeated in PBS containing $0.1 \%$ Triton $X-100$ for 15 minutes. The sections were blocked by $10 \%$ normal Donkey Serum for 1 hour, then Rabbit polyclonal to Laminin (Abcam, USA, ab11575) or anti-Pax-7 antibody (Servicebio, China, GB113190) and anti-P21 antibody (Servicebio, China, GB11153) with 10\% normal Donkey Serum were incubated. Then incubate at $4{ }^{\circ} \mathrm{C}$ overnight, wash with PBS three times, add secondary antibody (Invitrogen, USA), incubate at room temperature for one hour, and wash again. Use 2 $\mu \mathrm{M}$ DAPI (Servicebio, China, G1012) to observe the nucleus. TissueFAXS (TissueGnostics GmbH, Vienna 
Austria) with a Zeiss Axio Imager Z2 Microscope System at $\times 20$ magnification to acquire Immunofluorescence image.

\section{Western blot analysis}

Mice gastrocnemius were dissected after sacrificed. Western blots were performed and analyzed as previously described [22]. Antibodies used for Western blots were: Pax7(Abcam, USA, ab199010), actin (Abcam, USA, ab5694), Lamp2 (Abcam, USA, ab2563), B-actin (Abcam, USA, ab8226), Lc3B (Sigma, Germany, L7S43), P16 (Abcam, USA, ab51243), P53 (Cell Signaling Technology, USA, 1C12).

\section{Quantitative analysis and statistical analysis}

The cell density, nuclear area, cell area and antibody expression were quantified by Image J software (version 1.8.0, National Institutes of Health, USA). The specific operations are: open the picture with Image $\mathrm{J}$ software. Convert the picture to 2 levels in the RGB Stack. Furthermore, mark the stained area, and the area of all pictures is uniformly marked (Image $\rightarrow$ Adjust $\rightarrow$ Threshold). Then, set the measurement parameters. In the pop-up measurement parameter setting option box, check the required measurement parameters: Area; Mean; IntDen (Integrated Density); Area fraction, and click OK to confirm.

The last step is to select Analyze Particles under the current gear, export the results and enter them into Prism 8.0 software (GraphPad Software, La Jolla, CA, USA) for statistical analysis. All data are shown as means \pm S.E.M. The statistical significance of the differences between the two groups was determined using an unpaired two-tailed Student's t-test as indicated in the bar graph. A value of P®0.05 was considered to be statistically significant.

\section{Results}

\section{Clinical-grade hUC-MSCs improved muscle strength and restored skeletal muscle morphology both in SAMP8 mice and D-gal-induced ageing mice.}

Based on the detection of the cellular viability, morphology, differentiation potential and surface markers, the clinical-grade hUC-MSCs met the quality standards of MSCs [21, 22] (Suppl. 1). After treatment with hUC-MSCs, the behaviour features of AAS mouse models were tested by grip test and anti-fatigue test, which are core measures of sarcopenia [27]. The result suggested that compared with the SAMP8 mice with PBS injection (termed P8-PBS group), the hUC-MSCs intervened mice (termed P8-MSC group) exhibited enhanced grip strength and anti-fatigue abilities (Fig. 1b, c), similar to the SAMR1 mice (termed R1 group).

After the behaviour tests, the mouse models were euthanasia (Fig. 1a; Suppl. 2a). The gastrocnemius muscles were collected and performed with histopathological testes by cross section and vertical section [28]. To better characterise the muscle size on different treatment means, we performed H\&E staining on 
the cross section of gastrocnemius muscle and measured the area and diameter of muscle cells. Firstly, we found that the cross-sectional area and diameter were significantly reduced in the P8-PBS group, but were rescued in P8-MSC group. A similar treatment effect was observed in the D-gal-induced ageing model (Suppl. 2). These results demonstrated that muscle functions and morphology in AAS mouse models could be restored after the hUC-MSCs treatment.

\section{hUC-MSCs restored the ratio of slow and fast motor units of skeletal muscle in two mouse models.}

Skeletal muscle fibres can be divided into slow myosin (type I) fibres and fast myosin (type II) fibres [29], which has a critical role in accurately assessing the degree to which muscle fibres are affected in ageing muscle [30]. Age-related loss of muscle mass was primarily due to a decrease in the total number of slow and fast myosin fibres and secondarily to the preferential atrophy of fast myosin fibres [31-33]. In order to estimate the ability of hUC-MSCs to prevent the small decrease in fast fibres abundance, we observed the proportion of slow and fast myosin fibres in extensor digitorum longus (EDL). The result suggested, compared with the R1 group and P8-MSC group, the muscle cells of the P8-PBS group showed a more significant proportion of fast myosin fibres (Fig. 2). Likewise, the same phenomenon about muscle contraction could also be found in the D-gal-induced ageing model (Suppl. 3). These results demonstrated that hUC-MSC treatment increased the ratio of slow and fast motor units to enhance skeletal muscle performance.

\section{hUC-MSCs regulated the extracellular matrix of muscle cells in AAS mouse models.}

The extracellular matrix (ECM) plays a critical role in the growth of muscle cells by the establishment of the cellular niche and mediating the signal transduction [34]. With ageing, the ECM around muscle cells could also change, such as the reduced muscle fibre sarcolemma stability and weakened cell adhesion function [35]. In order to describe the changes in AAS models, we detected the expression of dystrophin and laminin, which played critical roles in ECM to stabilize the sarcolemma of muscle fibre and participate in cell communication [35-38]. The immunofluorescent images showed that the muscle cells in SAMP8 mice exhibited lower expression of dystrophin and laminin proteins expression than the SAMR1 mice. After the hUC-MSCs treatment, the expression of dystrophin and laminin were obviously increased, suggesting the ECM of muscle cells was restored (Fig. 3). Similarly, the elevated expressions of dystrophin and laminin in the D-gal-induced ageing model were also observed after hUC-MSCs treatment (Suppl. 4). The above results indicated that hUC-MSCs maintained muscle cell adhesion, improved their microenvironment and advanced muscle toughness and tensile strength in AAS by restoring the muscle ECM. 


\section{hUC-MSCs restrained the decline in the number of muscle satellite cells in two mouse models.}

It's known that the regenerative capacity of skeletal muscle declined with ageing, which is dominated by the dedicated muscle stem cells (MuSCs), also known as satellite cells. To decipher whether hUC-MSCs could prevent the depletion and exhaustion of MuSCs by improving its proliferation, we detected the expression of Pax7-the specific marker of MuSCs in skeletal muscle cells $[39,40]$. Fluorescence image and Western blot indicated that the quantity of Pax-7 $7^{+}$in the SAMP8 mice was lower than those in the SAMR1 mice. Nevertheless, ageing defects in the number of MuSCs were markedly recovered after hUCMSC treatment (Fig. 4). Meanwhile, intraperitoneal injection of hUC-MSCs restored the number of MuSCs and encouraged myogenesis in D-gal-induced ageing model (Suppl. 5). Results from two models suggested that after hUC-MSCs treatment, the number of MuSCs in AAS mouse models were significantly recovered, which could maintain the stability of the stem cell pool, or promote its proliferation and differentiation, remodel muscle fibres to ensure muscle regeneration.

\section{hUC-MSCs increased autophagy and delayed muscle cells senescence via p16-Rb/p53-p21 axis.}

It's known that senescence was closely related to cellular autophagy. Reduced autophagy could accelerate ageing, while increased autophagy has the potential of anti-ageing [41]. To investigate how hUC-MSCs could reverse the muscle dysfunctions in AAS mouse models via regulating muscle autophagy, we assessed the expression of Lamp2 [42] and LC3- II/I [43], which are autophagy-related biomarkers. Western blot images showed that compared with the ageing group, the expression of Lamp2 and LC3- II/I in the P8-MSC group (Fig. 5a, b) and the D-gal-MSC group (Suppl. 6a, b) increased enormously, indicating that autophagy was activated with the presence of hUC-MSCs.

Accelerated senescence [44] and inhibited autophagy [45] are associated with various pathways, such as inhibition of p53, p16 and p21 activation, among others. Western blot and immunofluorescence images showed that the expressions of p16, p53 and p21 were decreased in the P8-MSC group (Fig. 5c-e) and the D-gal-MSC group (Suppl. S6c-e) compared with the P8-PBS and the D-gal-PBS groups, suggesting hUCMSCs treatment could inhibit the activation of senescence-related pathways.

Collectively, hUC-MSCs played a crucial role in reconstructing myocyte autophagy to provide self-energy supply, and it could down-regulate the classic p16 / p53-p21 axis to delay myocyte ageing.

\section{Discussion}

AAS, the primary clinical disease of the elderly, has become a key challenge for healthy ageing [27]. It is estimated that approximately $5-13 \%$ of the 'healthy' elderly suffer from AAS [46-48]. Furthermore, AAS is essential to risk factors in disabling conditions, such as cognitive decline and osteoporosis $[6,49]$. 
However, there are no available therapies for AAS currently [50]. Therefore, as global ageing intensifies, it's urgent to propose safe and effective intervention strategies to avert the transition to disability and medical institutionalization of older individuals [9].

Recently, many studies, including ours, have confirmed that hUC-MSCs are an ideal stem cell for universal application, and that transplantation is an effective treatment for age-related degenerative diseases [51]. Further, the beneficial effects and effective utilization of hUC-MSCs in reducing muscle damage, enhancing tissue repair, and muscular regeneration had been reported in some muscle atrophy models, which might be based on their secretory capacity $[15-17,52,53]$. These studies suggested that hUCMSCs might bring new hope for the treatment of AAS.

Selecting and confirming better experimental models was a key part of this study. It's known that SAMP8 is currently the most commonly used SAMP strain to study AAS [27]. However, the phenotype of the D-galinduced ageing model in sarcopenia is unrevealed. Here, we first verified the D-gal-induced ageing model also exhibited typical AAS features (Suppl.2-6), so that it can be used as a supplementary model to screen the clinical treatment of sarcopenia comprehensively.

Here, we found that hUC-MSCs prevented muscle fibre atrophy and enhanced muscle strength and performance effectively (Fig. 1, see also Suppl.2). It can also impede the age-related loss of muscle mass owing to the decrease of MyHC fibres [54,55] and repair fast myosin fibres decrease in the EDL [56], which was evident in Fig. 2 and Suppl. 3. At the cellular level, hUC-MSCs effectively promoted the establishment of ECM (Fig. 3, and Suppl. 4) that provided a niche for signaling transduction between muscle cells $[57,58]$. When tissue injury occurred, hUC-MSCs induced the activation of the skeletal muscle satellite cells in ECM (Fig. 4, and Suppl. 5) and mobilized them to recapitulate the developmental pathway by adjusting their quantity[58]. Specifically, we found that hUC-MSCs up-regulated autophagy and rescued the cell senescence in AAS model via down-regulation of the p16 / p53-p21 axis (Fig. 5, and Suppl. 6), which inhibited the expression of CDK cyclin and Rb protein expression phosphorylation, reduced the accumulation of damaged proteins, provided energy for the activation of MuSCs, and enabled muscle regeneration when was needed [59-63]. Collectively, our results demonstrated that hUCMSCs transplantation could reverse skeletal muscle dysfunction from multiple aspects, including cellular components, cell structure and cell function, and ultimately restore muscle strength in AAS mice (Fig. 6).

All in all, to our knowledge, this is the first study on applying clinical-grade hUC-MSCs to AAS mouse models. All the data demonstrated that clinical-grade hUC-MSCs could effectively counteract the progression of muscle ageing in AAS mice and clarified the underlying mechanism of systematically targeting AAS therapy. More importantly, hUC-MSC-based therapy may be a promising and effective muscle protective candidate to prevent AAS and other progressive age-related muscle diseases.

\section{Abbreviations}

AAS: age-associated sarcopenia; BL: basal lamina; cGMP: current Good Manufacturing Practice; DAS: disease-associated sarcopenia; D-gal: D-galactose; ECM: extracellular matrix; EDL: extensor digitorum 
longus; H\&E: hematoxylin-eosin; hUC-MSCs: human umbilical cord-derived mesenchymal stem cells; MFS: muscle fibre sarcolemma; MSCs: Mesenchymal stem cells; MuSCs: muscle stem cells; NIFDC: National Institute of Food and Drug Control.

\section{Declarations}

\section{Acknowledgements}

We are grateful towards Quan Zeng for their methodical instructions. We thank Biao Zhang for excellent technical assistance.

\section{Funding}

This work was supported by grants from National Key Research and Development Program of China (2017YFA0103100 and 2017YFA0103103 to X.T.P.), Guangzhou Health Care and Cooperative Innovation Major Project (201604020009 to Y.L.J., 201803040005 to W.Y.) and The National Nature Science Foundation of China (81671385 to Y.L.J.)

\section{Data availability}

The data that support the findings of this study are available from the corresponding author upon reasonable request.

\section{Conflict of interests}

The authors indicate no potential conflicts of interest.

ORCID Wen Yue https://orcid.org/0000-0002-3935-360X Yali Jia https://orcid.org/0000-0002-2896-6021

\section{References}

1. Tournadre A, Vial G, Capel F, Soubrier M, Boirie Y (2019) Sarcopenia. Joint Bone Spine 86:309-314

2. Anker SD, Morley JE, von Haehling S (2016) Welcome to the ICD-10 code for sarcopenia. J Cachexia Sarcopenia Muscle 7:512-514

3. Bauer J, Morley JE, Schols A, Ferrucci L, Cruz-Jentoft AJ, Dent E et al (2019) Sarcopenia: A Time for Action. An SCWD Position Paper. J Cachexia Sarcopenia Muscle 10:956-961

4. Cruz-Jentoft AJ, Sayer AA (2019) Sarcopenia. Lancet 393:2636-2646

5. Cruz-Jentoft AJ, Landi F, Topinkova E, Michel JP (2010) Understanding sarcopenia as a geriatric syndrome. Curr Opin Clin Nutr Metab Care 13:1-7 
6. Pasco JA, Williams LJ, Jacka FN, Stupka N, Brennan-Olsen SL, Holloway KL et al (2015) Sarcopenia and the Common Mental Disorders: a Potential Regulatory Role of Skeletal Muscle on Brain Function? Curr Osteoporos Rep 13:351-357

7. Martinez BP, Batista AK, Gomes IB, Olivieri FM, Camelier FW, Camelier AA (2015) Frequency of sarcopenia and associated factors among hospitalized elderly patients. BMC Musculoskelet Disord $16: 108$

8. Giannoulis MG, Martin FC, Nair KS, Umpleby AM, Sonksen P (2012) Hormone replacement therapy and physical function in healthy older men. Time to talk hormones? Endocr Rev 33:314-377

9. Vinel C, Lukjanenko L, Batut A, Deleruyelle S, Pradere JP, Le Gonidec S et al (2018) The exerkine apelin reverses age-associated sarcopenia. Nat Med 24:1360-1371

10. Prockop DJ, Oh JY (2012) Mesenchymal stem/stromal cells (MSCs): role as guardians of inflammation. Mol Ther 20:14-20

11. Magatti D, Molteni M, Cardinali B, Rocco M, Ferri F (2017) Modeling of Fibrin Gels Based on Confocal Microscopy and Light-Scattering Data. Biophys J 112:410

12. Galderisi U, Giordano A (2014) The gap between the physiological and therapeutic roles of mesenchymal stem cells. Med Res Rev 34:1100-1126

13. Sandona M, Di Pietro L, Esposito F, Ventura A, Silini AR, Parolini O et al (2021) Mesenchymal Stromal Cells and Their Secretome: New Therapeutic Perspectives for Skeletal Muscle Regeneration. Front Bioeng Biotechnol 9:652970

14. Abrigo J, Rivera JC, Aravena J, Cabrera D, Simon F, Ezquer F et al (2016) High Fat Diet-Induced Skeletal Muscle Wasting Is Decreased by Mesenchymal Stem Cells Administration: Implications on Oxidative Stress, Ubiquitin Proteasome Pathway Activation, and Myonuclear Apoptosis. Oxid Med Cell Longev 2016:9047821

15. Kim MJ, Kim ZH, Kim SM, Choi YS (2016) Conditioned medium derived from umbilical cord mesenchymal stem cells regenerates atrophied muscles. Tissue Cell 48:533-543

16. Caplan Al (2007) Adult mesenchymal stem cells for tissue engineering versus regenerative medicine. J Cell Physiol 213:341-347

17. Park CM, Kim MJ, Kim SM, Park JH, Kim ZH, Choi YS (2016) Umbilical cord mesenchymal stem cellconditioned media prevent muscle atrophy by suppressing muscle atrophy-related proteins and ROS generation. In Vitro Cell Dev Biol Anim 52:68-76

18. Li TS, Shi H, Wang L, Yan C (2016) Effect of Bone Marrow Mesenchymal Stem Cells on Satellite Cell Proliferation and Apoptosis in Immobilization-Induced Muscle Atrophy in Rats. Med Sci Monit 22:4651-4660

19. Keating A (2012) Mesenchymal stromal cells: new directions. Cell Stem Cell 10:709-716

20. Zhao H, Feng J, Seidel K, Shi S, Klein O, Sharpe P et al (2014) Secretion of shh by a neurovascular bundle niche supports mesenchymal stem cell homeostasis in the adult mouse incisor. Cell Stem Cell 14:160-173 
21. Cao N, Liao T, Liu J, Fan Z, Zeng Q, Zhou J et al (2017) Clinical-grade human umbilical cord-derived mesenchymal stem cells reverse cognitive aging via improving synaptic plasticity and endogenous neurogenesis. Cell Death \& Disease 8:e2996

22. Jia Y, Cao N, Zhai J, Zeng Q, Zheng P, Su R et al (2020) HGF Mediates Clinical-Grade Human Umbilical Cord-Derived Mesenchymal Stem Cells Improved Functional Recovery in a SenescenceAccelerated Mouse Model of Alzheimer's Disease. Advanced Science 7:1903809

23. Kim MJ, Kim ZH, Kim SM, Choi YS (2016) Conditioned medium derived from umbilical cord mesenchymal stem cells regenerates atrophied muscles. Tissue Cell 48:533-543

24. Park CM, Kim MJ, Kim SM, Park JH, Kim ZH, Choi YS (2016) Umbilical cord mesenchymal stem cellconditioned media prevent muscle atrophy by suppressing muscle atrophy-related proteins and ROS generation. In Vitro Cell Dev Biol Anim 52:68-76

25. Kim MJ, Kim ZH, Kim SM, Choi YS (2016) Conditioned medium derived from umbilical cord mesenchymal stem cells regenerates atrophied muscles. Tissue Cell 48:533-543

26. Byrne AB, Garcia AG, Brahamian JM, Mauri A, Ferretti A, Polack FP et al (2021) A murine model of dengue virus infection in suckling C57BL/6 and BALB/c mice. Animal Model Exp Med 4:16-26

27. Xie WQ, He M, Yu DJ, Wu YX, Wang XH, Lv S et al (2021) Mouse models of sarcopenia: classification and evaluation. J Cachexia Sarcopenia Muscle 12:538-554

28. Manini TM, Clark BC (2016) Letter to the editor: results from a Web-based survey to identify dynapenia screening tools and risk factors. J Cachexia Sarcopenia Muscle 7:499-500

29. Scott W, Stevens J, Binder-Macleod SA (2001) Human skeletal muscle fiber type classifications. Phys Ther 81:1810-1816

30. Murach KA, Dungan CM, Kosmac K, Voigt TB, Tourville TW, Miller MS et al (2019) Fiber typing human skeletal muscle with fluorescent immunohistochemistry. J Appl Physiol (1985) 127:1632-1639

31. Roos MR, Rice CL, Vandervoort AA (1997) Age-related changes in motor unit function. Muscle Nerve 20:679-690

32. Lexell J, Taylor CC, Sjostrom M (1988) What is the cause of the ageing atrophy? Total number, size and proportion of different fiber types studied in whole vastus lateralis muscle from 15- to 83-yearold men. J Neurol Sci 84:275-294

33. Andersen JL (2003) Muscle fibre type adaptation in the elderly human muscle. Scand J Med Sci Sports 13:40-47

34. Carmignac V, Durbeej M (2012) Cell-matrix interactions in muscle disease. J Pathol 226:200-218

35. Labat-Robert J (2004) Cell-matrix interactions in aging: role of receptors and matricryptins. Ageing Res Rev 3:233-247

36. Koenig M, Monaco AP, Kunkel LM (1988) The complete sequence of dystrophin predicts a rodshaped cytoskeletal protein. Cell 53:219-228

37. Haenggi T, Fritschy JM (2006) Role of dystrophin and utrophin for assembly and function of the dystrophin glycoprotein complex in non-muscle tissue. Cell Mol Life Sci 63:1614-1631 
38. Hunter DD, Porter BE, Bulock JW, Adams SP, Merlie JP, Sanes JR (1989) Primary sequence of a motor neuron-selective adhesive site in the synaptic basal lamina protein S-laminin. Cell 59:905-913

39. Blau HM, Cosgrove BD, Ho AT (2015) The central role of muscle stem cells in regenerative failure with aging. Nat Med 21:854-862

40. Seale P, Asakura A, Rudnicki MA (2001) The potential of muscle stem cells. Dev Cell 1:333-342

41. Madeo F, Tavernarakis N, Kroemer G (2010) Can autophagy promote longevity? Nat Cell Biol 12:842846

42. Cuervo AM, Wong E (2014) Chaperone-mediated autophagy: roles in disease and aging. Cell Res 24:92-104

43. Sun Y, Berleth N, Wu W, Schlutermann D, Deitersen J, Stuhldreier F et al (2021) Fin56-induced ferroptosis is supported by autophagy-mediated GPX4 degradation and functions synergistically with mTOR inhibition to kill bladder cancer cells. Cell Death Dis 12:1028

44. Munoz-Espin D, Serrano M (2014) Cellular senescence: from physiology to pathology. Nat Rev Mol Cell Biol 15:482-496

45. Rubinsztein DC, Marino G, Kroemer G (2011) Autophagy and aging. Cell 146:682-695

46. Sepulveda-Loyola W, Osadnik C, Phu S, Morita AA, Duque G, Probst VS (2020) Diagnosis, prevalence, and clinical impact of sarcopenia in COPD: a systematic review and meta-analysis. J Cachexia Sarcopenia Muscle 11:1164-1176

47. Rolland Y, Czerwinski S, Abellan VKG, Morley JE, Cesari M, Onder G et al (2008) Sarcopenia: its assessment, etiology, pathogenesis, consequences and future perspectives. $J$ Nutr Health Aging $12: 433-450$

48. Cruz-Jentoft AJ, Baeyens JP, Bauer JM, Boirie Y, Cederholm T, Landi F et al (2010) Sarcopenia: European consensus on definition and diagnosis: Report of the European Working Group on Sarcopenia in Older People. Age Ageing 39:412-423

49. Papachristou E, Ramsay SE, Lennon LT, Papacosta O, lliffe S, Whincup PH et al (2015) The relationships between body composition characteristics and cognitive functioning in a populationbased sample of older British men. BMC Geriatr 15:172

50. Cohen S, Nathan JA, Goldberg AL (2015) Muscle wasting in disease: molecular mechanisms and promising therapies. Nat Rev Drug Discov 14:58-74

51. Reyhani S, Abbaspanah B, Mousavi SH (2020) Umbilical cord-derived mesenchymal stem cells in neurodegenerative disorders: from literature to clinical practice. Regen Med 15:1561-1578

52. Wang QQ, Jing XM, Bi YZ, Cao XF, Wang YZ, Li YX et al (2018) Human Umbilical Cord Wharton's Jelly Derived Mesenchymal Stromal Cells May Attenuate Sarcopenia in Aged Mice Induced by Hindlimb Suspension. Med Sci Monit 24:9272-9281

53. Sandona M, Di Pietro L, Esposito F, Ventura A, Silini AR, Parolini O et al (2021) Mesenchymal Stromal Cells and Their Secretome: New Therapeutic Perspectives for Skeletal Muscle Regeneration. Front Bioeng Biotechnol 9:652970 
54. Larsson L, Ansved T (1995) Effects of ageing on the motor unit. Prog Neurobiol 45:397-458

55. Sullivan VK, Powers SK, Criswell DS, Tumer N, Larochelle JS, Lowenthal D (1995) Myosin heavy chain composition in young and old rat skeletal muscle: effects of endurance exercise. J Appl Physiol (1985) 78:2115-2120

56. Messa G, Piasecki M, Hill C, McPhee JS, Tallis J, Degens H (2019) Morphological alterations of mouse skeletal muscles during early ageing are muscle specific. Exp Gerontol 125:110684

57. Scott RW, Arostegui M, Schweitzer R, Rossi F, Underhill TM (2019) Hic1 Defines Quiescent Mesenchymal Progenitor Subpopulations with Distinct Functions and Fates in Skeletal Muscle Regeneration. Cell Stem Cell 25:797-813

58. Uezumi A, Ikemoto-Uezumi M, Zhou H, Kurosawa T, Yoshimoto Y, Nakatani M et al (2021) Mesenchymal Bmp3b expression maintains skeletal muscle integrity and decreases in age-related sarcopenia. J Clin Invest 131

59. Kim WY, Sharpless NE (2006) The regulation of INK4/ARF in cancer and aging. Cell 127:265-275

60. Gil J, Peters G (2006) Regulation of the INK4b-ARF-INK4a tumour suppressor locus: all for one or one for all. Nat Rev Mol Cell Biol 7:667-677

61. Halazonetis TD, Gorgoulis VG, Bartek J (2008) An oncogene-induced DNA damage model for cancer development. Science 319:1352-1355

62. Sousa-Victor $P$, Garcia-Prat $L$, Munoz-Canoves $P$ (2021) Control of satellite cell function in muscle regeneration and its disruption in ageing. Nat Rev Mol Cell Biol

63. Garcia-Prat L, Martinez-Vicente M, Perdiguero E, Ortet L, Rodriguez-Ubreva J, Rebollo E et al (2016) Autophagy maintains stemness by preventing senescence. Nature 529:37-42

\section{Figures}


a

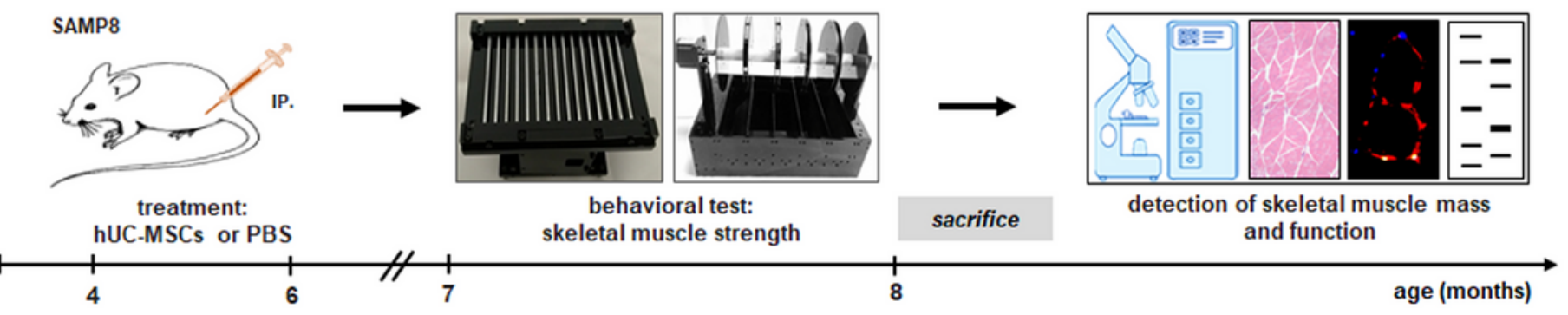

b

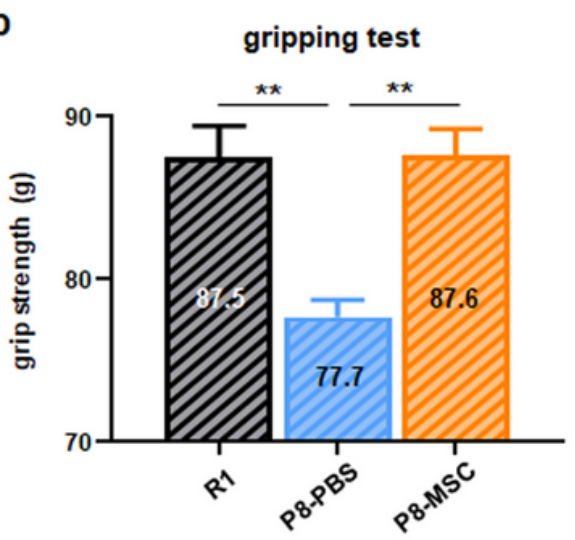

C

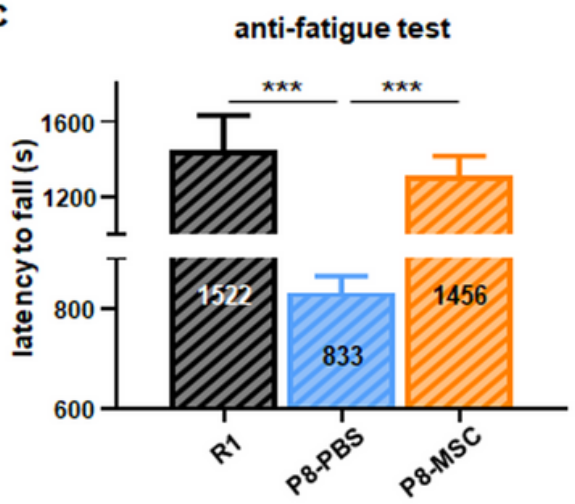

d

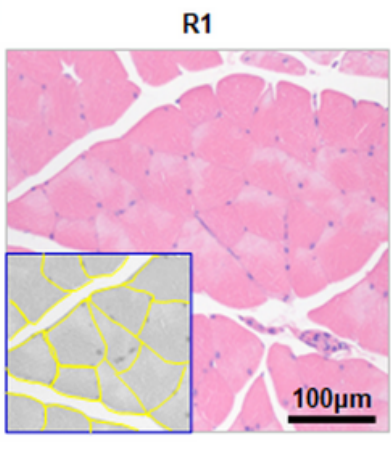

e

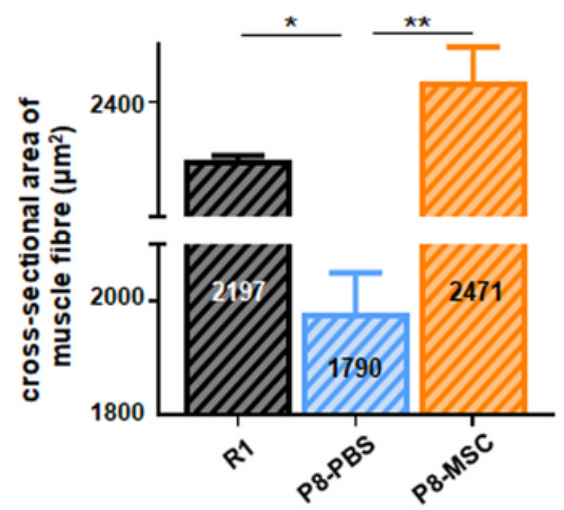

P8-PBS
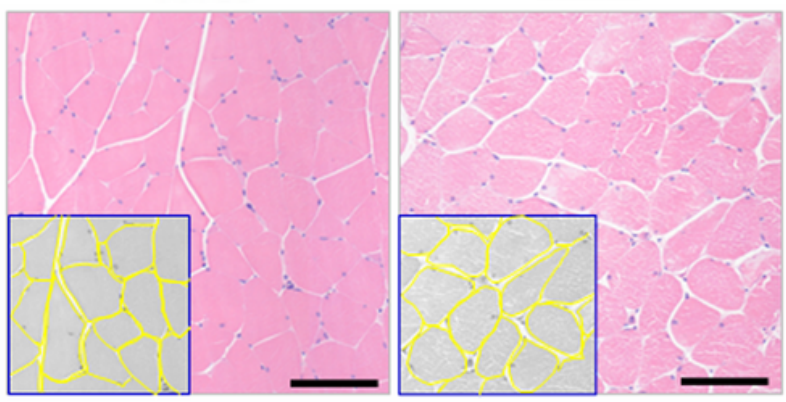

f

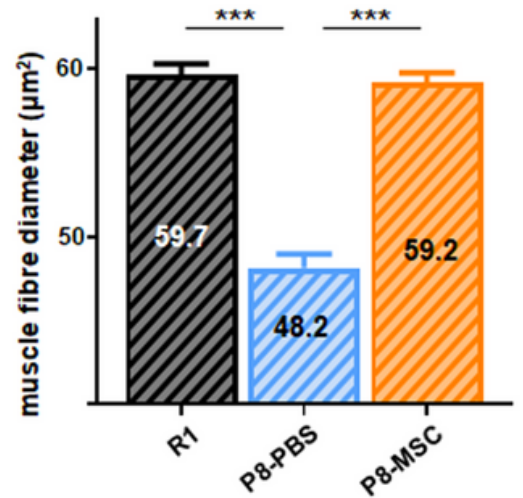

\section{Figure 1}

Clinical-grade hUC-MSCs improved muscle strength and restored skeletal muscle morphology in SAMP8 mice. a Schematic illustrating the chronological order used for hUC-MSCs or PBS treatment, behavioural tests, immunostaining, western blotting and others. (b, c) Gripping and anti-fatigue were tested to evaluate muscle strength. The time of Latency to fall in the Rota Rod system was used to reflect the muscle endurance, and gripping test was used to show the grip strength of SAMP8 mice $(n=6-7$ per group). $d$ The representative cross-sections of gastrocnemius muscle were stained with H\&E to observe better the morphology of muscle cells in R1, P8-PBS, and P8-MSC groups (scale bar=100 $\mu \mathrm{m})$. (e, f) Quantitative analysis of muscle fiber cross-sectional area $\left(\mu \mathrm{m}^{2}\right)$ and muscle fiber diameter $\left(\mu \mathrm{m}^{2}\right)$ in SAMP8 mice ( $n=3$ views per group; all data shown as mean $\pm S E M, * P<0.05, * \star P<0.01, * \star * P<0.001$ ). 

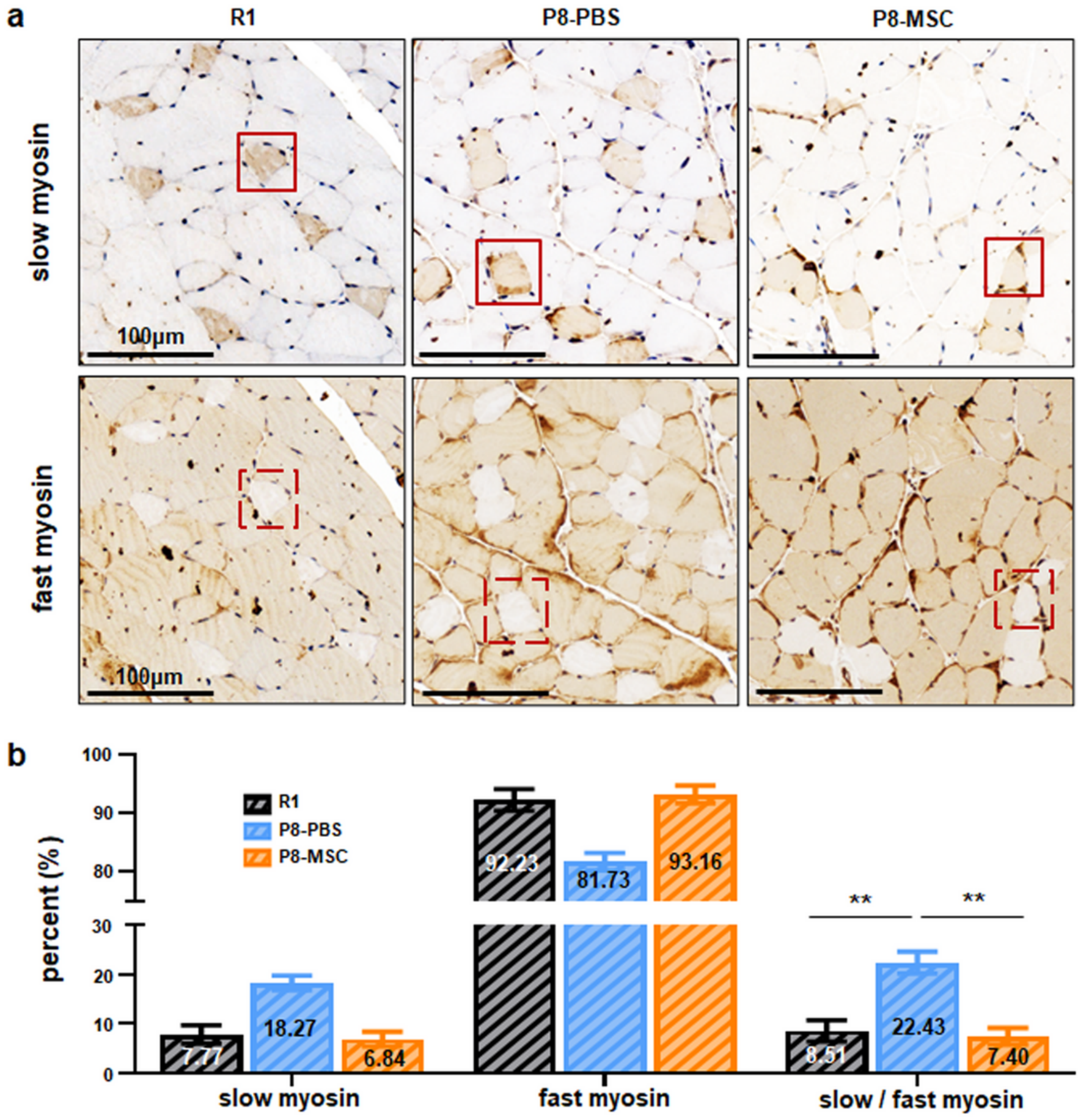

Figure 2

hUC-MSCs restored the ratio of slow and fast motor units of skeletal muscle in SAMP8 mice. a Representative immunohistochemical images of extensor digitorum longus (EDL) muscle cells showed the location of fast myosin and slow myosin in R1, P8-PBS and P8-MSC mice. Inside the square is the same muscle cell (scale bar $=100 \mu \mathrm{m}$ ). b The percent of fast myosin and slow myosin in EDL muscle cells of SAMP8 mice ( $n=2$ or 3 views per group; all data shown as mean $\pm S E M, * P<0.05,{ }^{*} P<0.01, \star \star \star P<$ $0.001)$. 

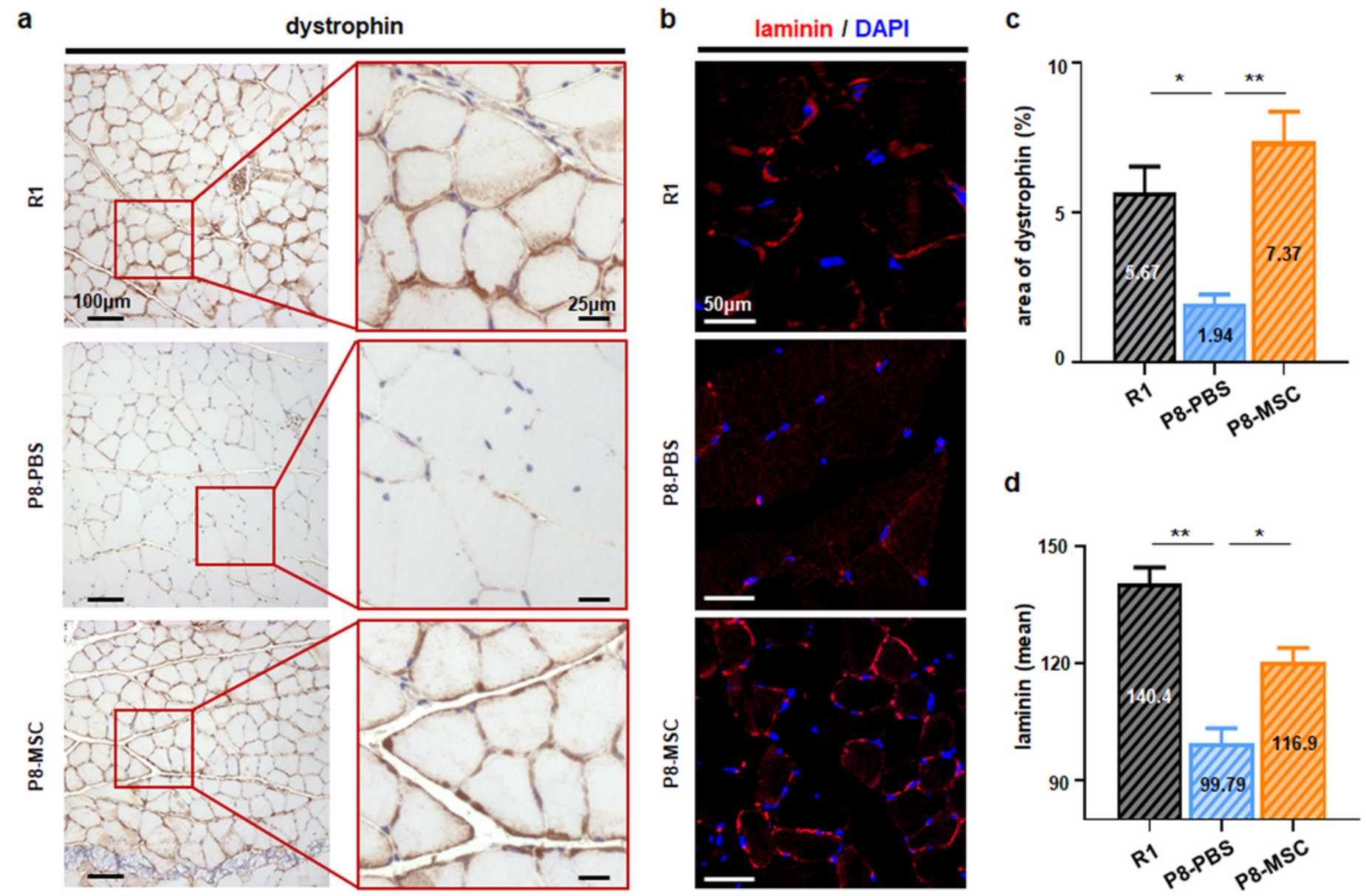

Figure 3

hUC-MSCs modulated the expression of important extracellular matrix proteins in SAMP8 mice. a Representative immunohistochemical images of ECM showed dystrophin protein expression in the SAMP8 mouse model (scale bar=100 $\mu \mathrm{m}$ ). Individual cells expressing positive protein under high magnification are shown in the square (scale bar=25 $\mu \mathrm{m}$ ). c The percentage of dystrophin between different groups in the visual field area was quantified ( $n=3$ views per group). $b$ Representative immunohistochemistry images indicated Laminin protein expression in SAMP8 mice (scale bar=50 $\mu \mathrm{m}$ ). $d$ The average fluorescence value of Laminin protein expression was quantified according to the random visual field in different SAMP8 mice groups ( $n=2$ or 3 views per group; all data shown as mean $\pm S E M$, * $P$ $<0.05, \star \star P<0.01, * \star * P<0.001)$. 
a
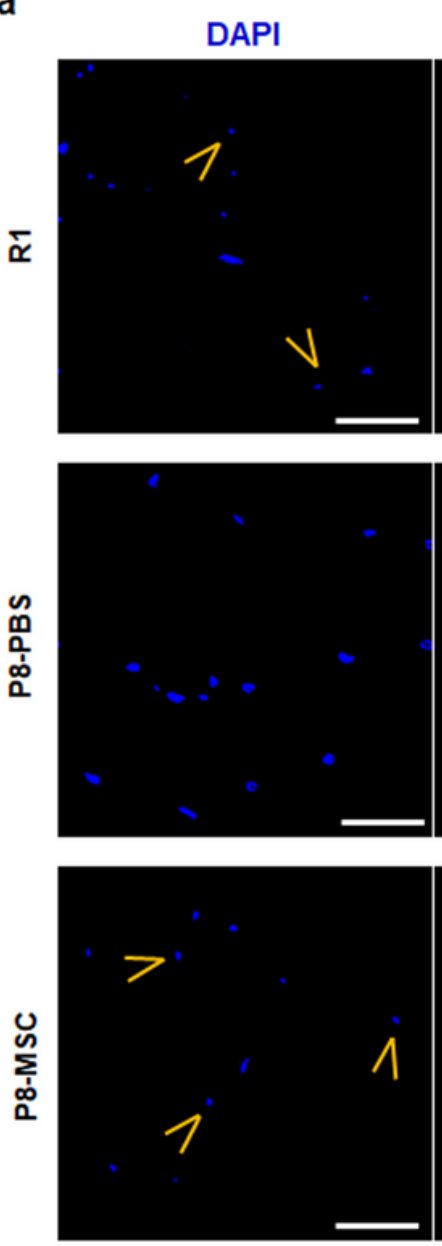

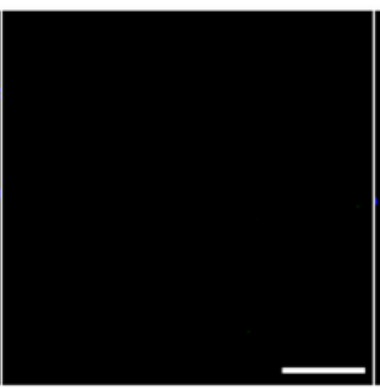

Pax-7
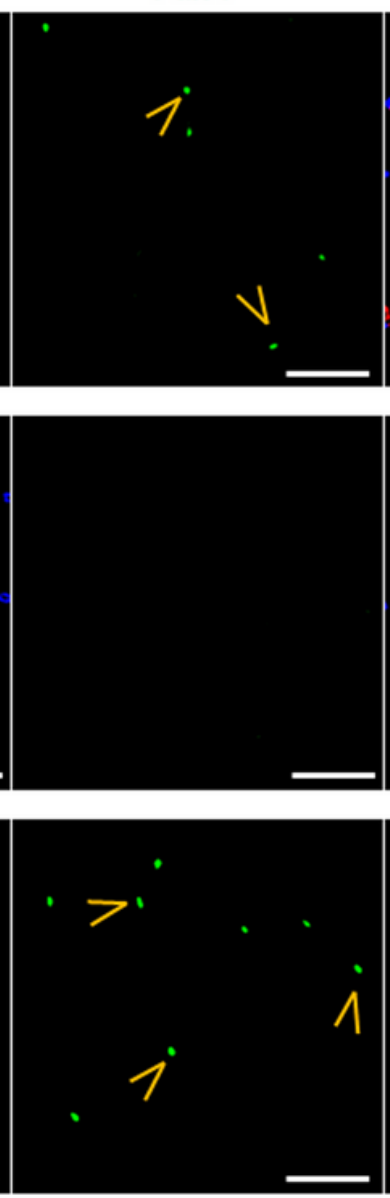

Pax-7/laminin /DAPI
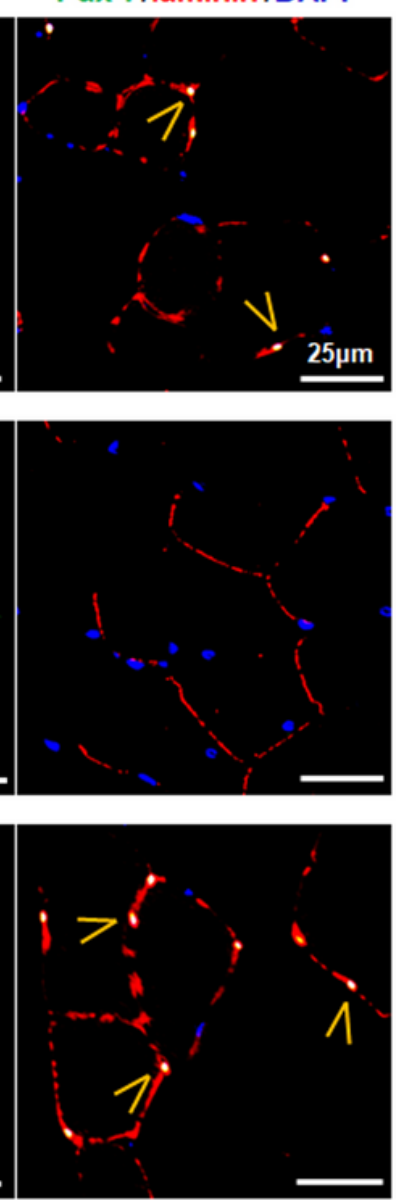

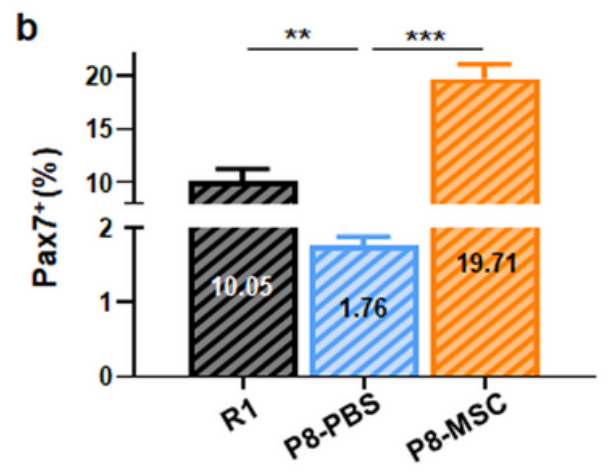

C

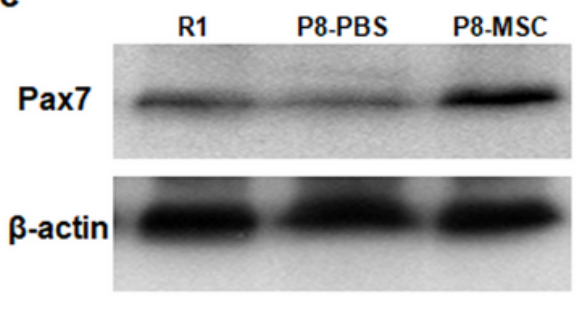

d

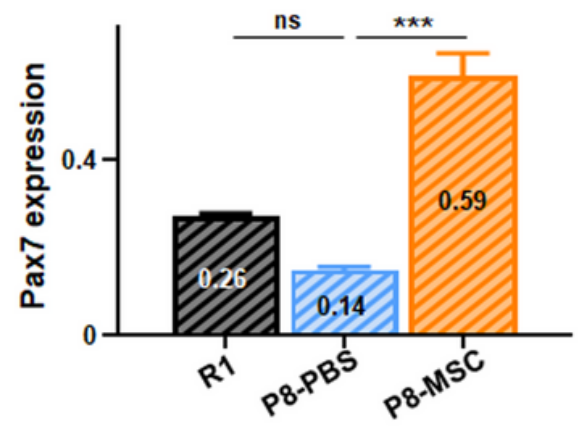

Figure 4

hUC-MSCs restrained the decline in the number of muscle satellite cells in SAMP8 mice. $(a, b)$ Representative immunohistochemical images of satellite cells in skeletal muscle showed the expression of Pax-7, and the number of Pax- $7^{+}$cells in different visual fields in SAMP8 mice was calculated (scale bar $=25 \mu \mathrm{m} ; \mathrm{n}=3$ views per group). (c, d) The expression of Pax-7 in gastrocnemius muscle of R1, P8-PBS and P8-MSC groups were detected by western blot and statistically analyzed ( $\mathrm{n}=3$ per group; all data shown as mean $\pm \mathrm{SEM},{ }^{*} \mathrm{P}<0.05,{ }^{\star *} \mathrm{P}<0.01$, $\left.{ }^{\star \star *} \mathrm{P}<0.001\right)$. 
a
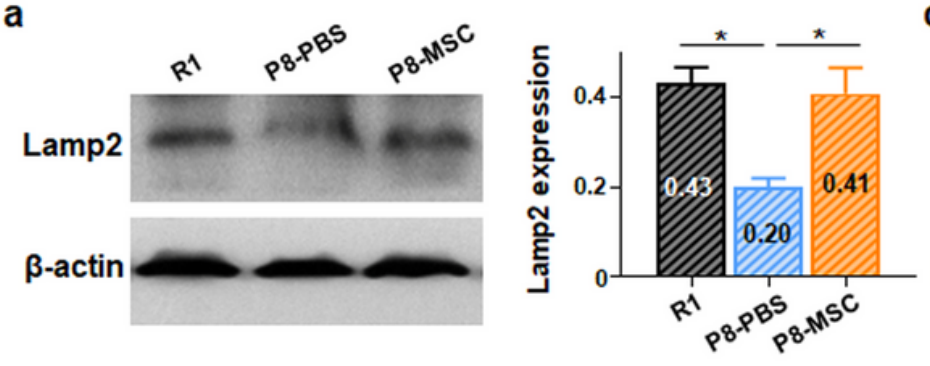

b

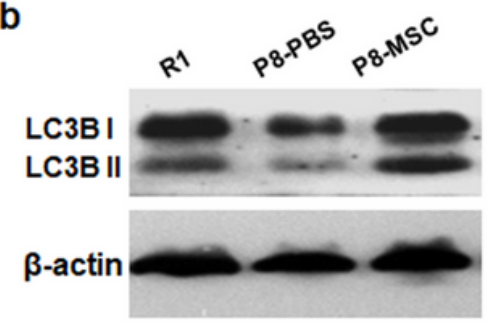

e

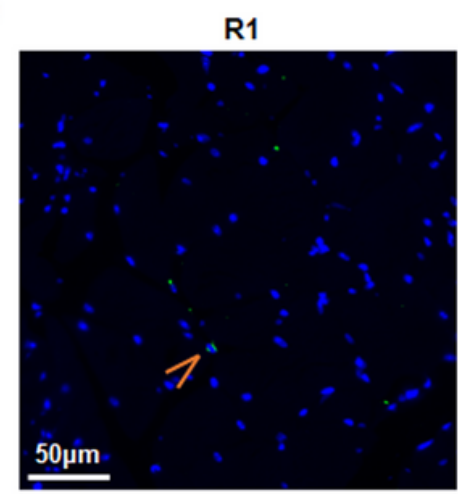

p21/DAPI

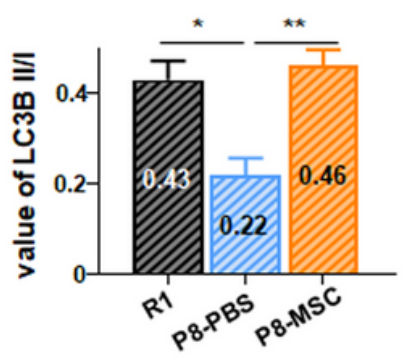

P8-PBS
C

p16
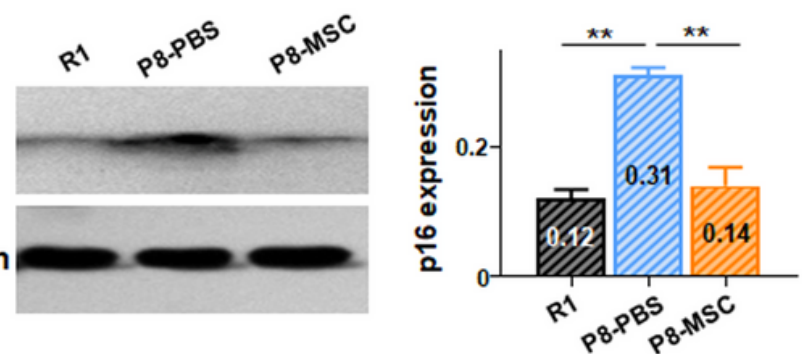

d

$p^{1} \quad p^{8^{\beta^{S}}} p^{8^{N^{S C}}}$

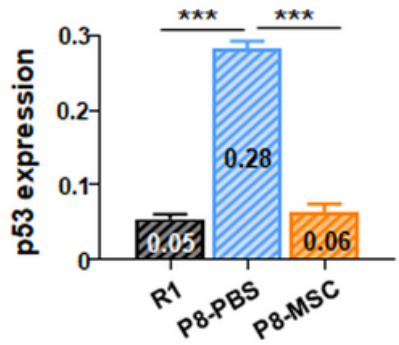

p53

$\beta$-actin
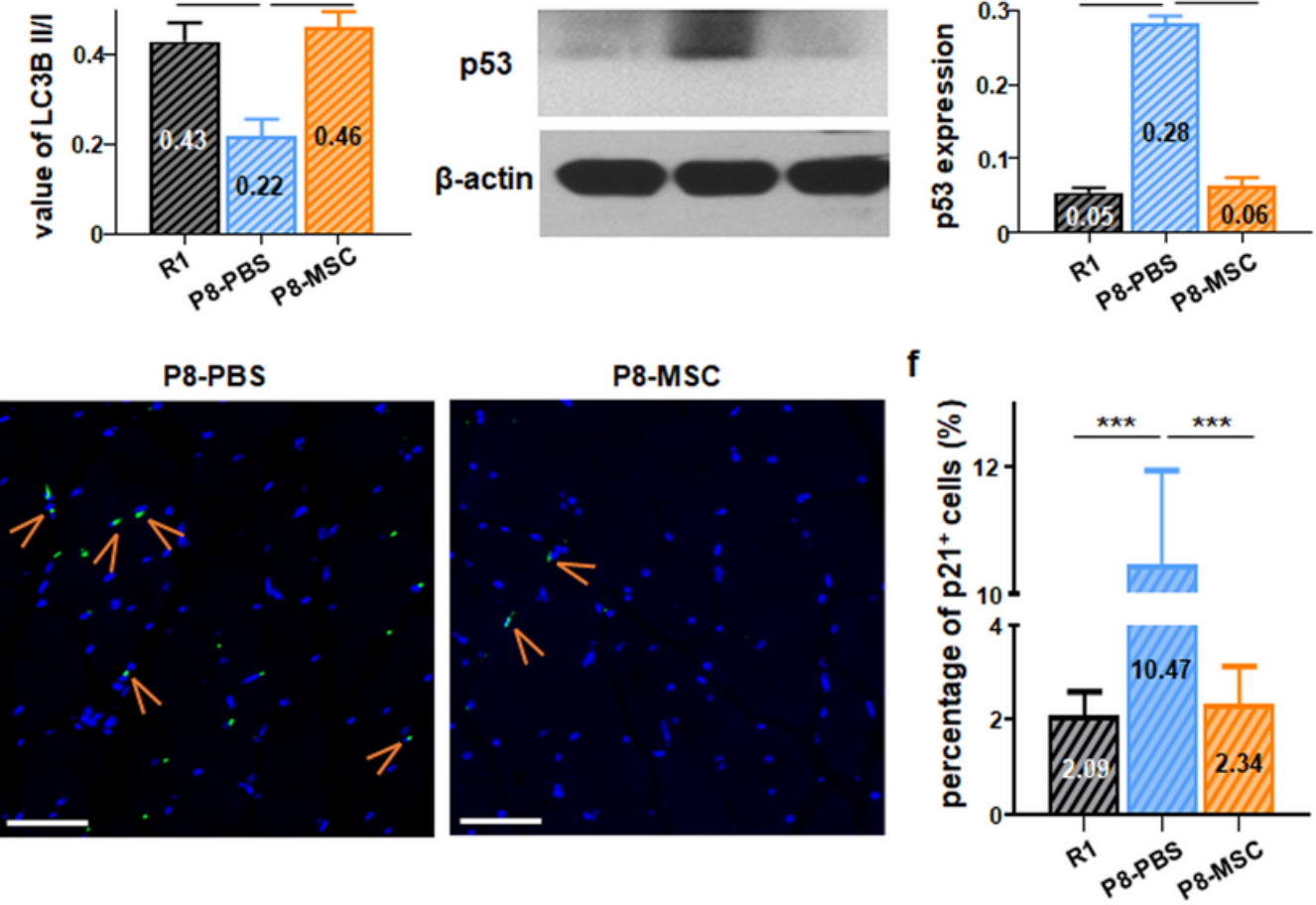

\section{Figure 5}

hUC-MSCs increased autophagy and delayed myocyte senescence through p16-Rb / p53-p21 axis in SAMP8 mice. (a-d) The expressions of Lamp2, LC3B II/I, p16 and p53 in muscle cells of R1, P8-PBS and P8-MSC mice were detected by western blot ( $n=3$ per group; all data shown as mean $\pm S E M$, ${ }^{\star} P<0.05$, $* * P$ $<0.01)$. (e, f) Representative immunofluorescence images showed the expression of a signal pathway p21 in skeletal muscle in R1, P8-PBS and P8-MSC mice (scale bar $=50 \mu \mathrm{m}$ ) and quantified its expression according to the mean fluorescence value in different perspectives of view $(n=5-6$ views per group; all data shown as mean $\pm \mathrm{SEM}, * * * \mathrm{P}<0.001)$. 

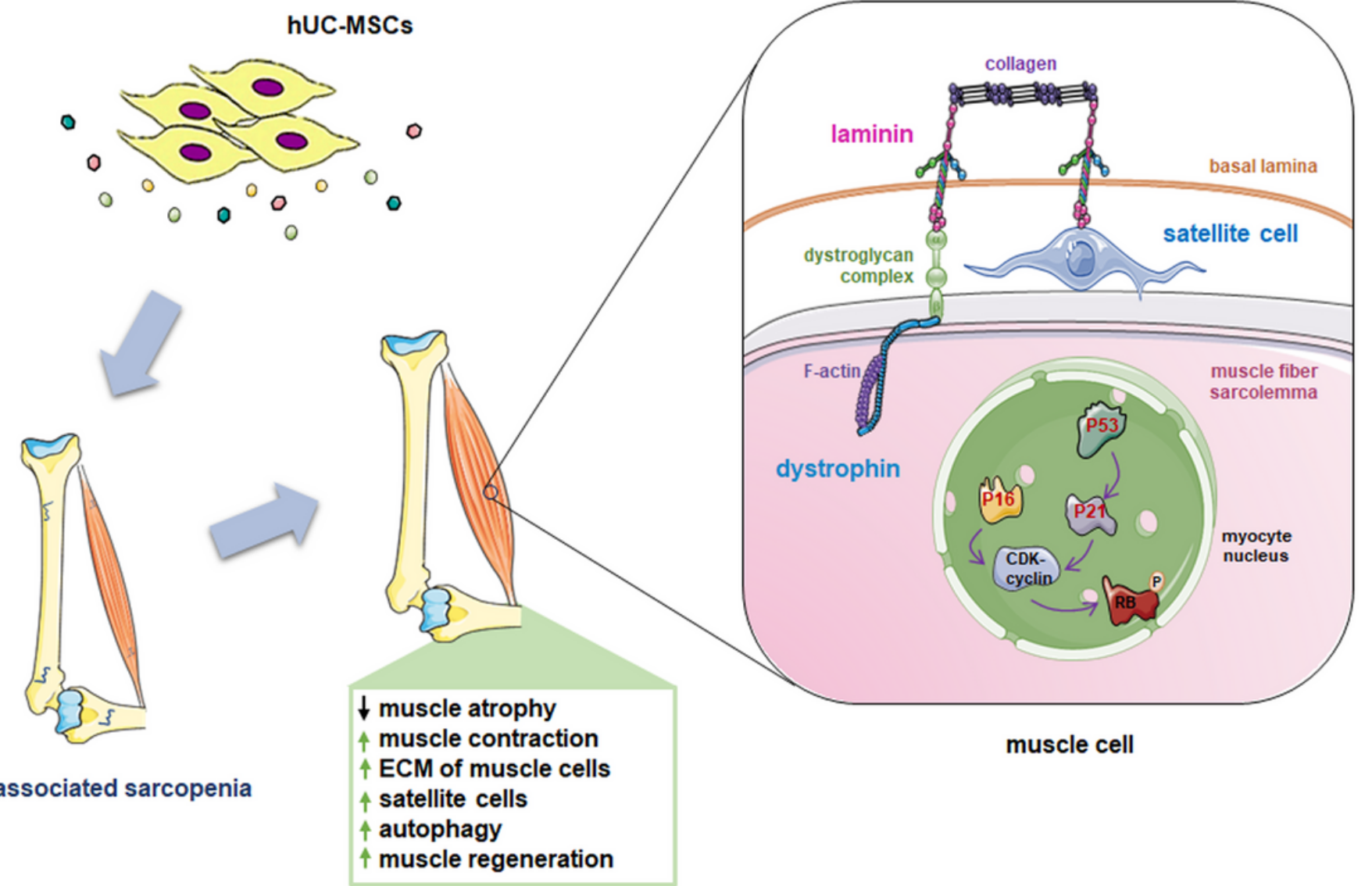

\section{Figure 6}

Schematic diagram of hUC-MSCs treatment in age-associated sarcopenia. hUC-MSCs secrete multiple bioactive molecules to the skeletal muscle cells of AAS mice through the secretome. In muscle cells, satellite cells are located between the basal lamina (BL) and the muscle fibre sarcolemma (MFS), where they interact with the matrix components of the niche. Satellite cells expressing Pax-7 at rest bind to collagen and laminin. In addition, MFS is connected to BL through the dystroglycan complex and laminin, and the dystrophin complex is combined with the F-actin cytoskeleton through dystrophin. In the muscle nucleus, the secretory molecules of hUC-MSCs promote muscle autophagy and slow down ageing by down-regulating p53 and p53 downstream molecules p21 and p16.

\section{Supplementary Files}

This is a list of supplementary files associated with this preprint. Click to download.

- Suppl.1.tiff

- Suppl.2.tiff

- Suppl.3.tiff 
- Suppl.4.tiff

- Suppl.5.tiff

- Suppl.6.tiff

Page 21/21 